\title{
流形上离散动力系统周期轨发生 各种分歧的充要条件
}

\author{
张广远 \\ （中国科学院成都分院数理科学研究室）
}

记号说明

$M-n$ 维光滑流形, $\|\cdot\|-R^{n}$ 中的模, $1 \cdot 1-R^{n}$ 上线性映射的模, $B_{0}-R^{n}$ 中单位 闭球, $\|\cdot\|_{C^{1}-C^{r}}\left(B_{0}, B_{0}\right)$ 中的 $C^{1}$ 模 $(r \geqslant 1), U_{X}(\varepsilon)-U_{X}(\varepsilon)=\left\{y \in B_{0} \mid\|y-x\|<\right.$ $\varepsilon\} V_{f}(\delta)-f \in C^{r}\left(B_{0}, B_{0}\right)$ 的 $C^{1}$ 模意义下的 $\delta$-邻域。

$f^{k}-f$ 的 $k$ 次迭代. $f^{0}=i d, f^{k+1}=f \circ f^{k}$

本文中矩阵的特征根概指复数域上的特征根。

\section{一、引立及本文结果}

倍周期分歧 ${ }^{[, 2]}$ 是近年来动力系统的一个重要方向, 与目前动力系统的一些重要课题如 Feigenbaum 现象 ${ }^{[3,4]}$ 及 Chaos ${ }^{[5,6]}$ 密切相关. 目前对分歧的研究大多限于低维上具体的含参系 统,本文引进一般情形下周期轨分歧的定义,得到了高维流形上较完整的结果.

定义 1 设 $f \in C^{r}(M, M)(r \geqslant 1), X_{0} \in \operatorname{Per}(f, k), m \in Z^{+}$, 在 $X_{0}$ 的某一邻域 $U_{X_{0}} \subset M$ 里无 $f$ 的异于 $X_{0}$ 的 $m k$ 周期点, 但对 $X_{0}$ 的任一邻域 $U$, 存在 $f$ 在任意小 $C^{1}$ 扰动下的 $g$, 使 得 $g$ 在 $U$ 里至少有两个 $m k$ 周期点, 则称 $X_{0}$ 是 $f$ 的 $m$ 分歧周期点, $X_{0}$ 所在的周期轨是 $m$ 分 歧周期轨, $m$ 为分歧倍数.

由文献 [2], 一维自映射周期轨至多能发生 2 分歧,而在高维情形下,分歧复杂得多,任一 自然数都可以成为某一周期轨的分歧倍数，为此本文给出了发生各种倍数分歧的充分必要条 件(定理 1). 另有别于一维, 在高维情形下, 同一周期轨的分歧倍数不一定唯一, 本文又给出 了周期轨分歧倍数个数的最佳上界(定理 2).

定义 2 设 $f \in C^{r}(M, M), X_{0} \in \operatorname{Per}(f, k)$, 如果 $X_{0}$ 共有 $s$ 个分歧倍数, 则称 $X_{0}$ 是具 有 $s$ 种分歧方式的分歧周期轨，记以

$$
B_{f}^{n}\left(X_{0}\right)=s, \quad n=\operatorname{dim} M .
$$

定理 1 设 $f \in C^{r}(M, M), X_{0} \in \operatorname{Per}(f, k), m \in Z^{+}$, 在 $X_{0}$ 附近无 $f$ 的异于 $X_{0}$ 的 $m k$ 周期点, 则 $X_{0}$ 为 $\dot{m}$ 分歧周期点的充要条件是:

存在环绕 $X_{0}$ 的一个卡 $(U, h)$, 使得 $\left.D\left(h \circ f^{k} \circ h^{-1}\right)\right|_{b\left(X_{0}\right)}$ 有满足如下条件的一组 特征根 $\lambda_{1}, \lambda_{2}, \cdots, \lambda_{s}$.

i) $\lambda_{i}^{m}=1,1 \leqslant i \leqslant s$;

ii) $\forall p \in Z^{+}, p<m \Rightarrow \exists i, 1 \leqslant i \leqslant s, \exists \cdot \lambda_{i}^{p} \neq 1$,

本文 1986 年11月10日收到。 
推论 设 $f \in C^{\prime}(M, M), X_{0} \in \operatorname{Per}(f, k), m_{1}, m_{2} \in Z^{+}, X_{0}$ 同时是 $m_{1}, m_{2}$ 分歧周期点， 又设 $m$ 是 $m_{1}$ 和 $m_{2}$ 的最小公倍数，在 $X_{0}$ 附近无 $f$ 的异于 $X_{0}$ 的 $m k$ 周期点, 则 $X_{0}$ 是 $f$ 的 $m$ 分 歧周期点.

定理 2 设 $f \in C^{r}(M, M), X_{0} \in \operatorname{Per}(f, k)$, 则

$$
\begin{aligned}
& B_{f}^{n}\left(X_{0}\right) \leqslant\left\{\begin{array}{l}
2^{\frac{n}{2}}, \text { 当 } n=\operatorname{dim} M \text { 为偶数 } \\
2^{\frac{n+1}{2}}-1, \text { 当 } n=\operatorname{dim} M \text { 为奇数. }
\end{array}\right. \\
& \text { 二、几个引引理 }
\end{aligned}
$$

引玨 1 分歧性是局部 $C^{r}$ 共轭不变性. 证略.

引理 2 设 $f \in C^{r}\left(B_{o}, B_{o}\right), O \in F i x(f),\left.D f\right|_{o}=J$, 又设 $g \in C^{r}\left(B_{o}, B_{o}\right), G(X)=$ $g(X)-X$, 则对 $\forall m \in Z^{+}, \forall \eta>0, \exists \delta>0, \varepsilon>0$, 使得当 $g \in V_{f}(\delta), X \in U_{0}(\varepsilon)$ 时有

$$
\left\|g^{m}(X)-X-\left(\sum_{i=0}^{m-1} J^{i}\right)(G(X))\right\| \leqslant \eta\|G(X)\|
$$

( $O$ 是 $R^{n}$ 的原点， $B$ 。的中心. 这里将矩阵、微分、线性映射等同起来,以下同).

证 对 $m$ 进行归纳,

当 $m=1$ 时,结论显然.

假定当 $m=l$ 时结论成立, 即对 $\forall \eta>0, \exists \delta>0, \varepsilon>0$, 使得当 $g \in V_{f}(\delta), X \in U_{0}(\varepsilon)$ 时, (*) 对 $m=l$ 成立. 易见, $\exists \delta^{\prime}>0, \varepsilon^{\prime}>0, L>0$ 且 $\delta^{\prime}<\delta$, 使得当 $g \in V_{f}\left(\delta^{\prime}\right)$, $X \in U_{o}\left(\varepsilon^{\prime}\right)$ 时, $g(X) \in V_{o}(\varepsilon),|D G(X)|<L,|D(g-J)(X)| \leqslant \eta$, 因而由归纳假设, 对 $\forall X \in U_{o}\left(\varepsilon^{\prime}\right), g \in U_{f}\left(\delta^{\prime}\right)$, 有

$$
\begin{aligned}
& \left\|g^{l+1}(X)-X-\left(\sum_{i=0}^{l} J^{i}\right)(G(X))\right\| \\
= & \| g^{l+1}(X)-g(X)-\left(\sum_{i=0}^{l-1} J^{i}\right)(G(g(X)))+\left(\sum_{i=0}^{l-1} J^{i}\right)(G(g(X))) \\
& -\left(\sum_{i=1}^{l} J^{i}\right)(G(X))+g(X)-X-G(X) \| \\
\leqslant & \left\|g^{l}(g(X))-g(X)-\left(\sum_{i=0}^{l-1} J^{i}\right)(G(g(X)))\right\|+\left\|\sum_{i=0}^{l-1}\left(J^{i}(G(g(X))-J(G(X)))\right)\right\| \\
\leqslant & \eta\|G(g(X))\|+\left(\sum_{i=0}^{l-1}\left|J^{i}\right|\right) \cdot\|G(g(X))-J(G(X))\| \\
\leqslant & \eta\|G(g(X))-G(X)\|+\eta\|G(X)\|+L_{1}\|g(g(X))-g(X)-J(g(X))+J(X)\| \\
\stackrel{0}{\leqslant} & \eta L\|g(X)-X\|+\eta\|G(X)\|+L_{1}\|(g-J)(g(X))-(g-J)(X)\| \\
\stackrel{0}{\leqslant} & \eta L\|G(X)\|+\eta\|G(X)\|+L_{1} \eta\|g(X)-X\| \\
= & \left(1+L+L_{1}\right) \eta\|G(X)\|,
\end{aligned}
$$

其中 $L_{1}=\sum_{i=0}^{l-1}\left|J^{i}\right|$. (1)、(2)用了中值公式. 
由 $\eta$ 的任意性,归纳完成。证毕.

引理 3 设 $H$ 是 $n \times n$ 非退化矩阵, 则存在 $\eta_{0}>0$, 使得对 $\forall x \in R^{*}$, 有

$$
X \neq 0 \Rightarrow\|H(X)\|>\eta_{0}\|X\| \text {. 证略. }
$$

引理 4 设 $J$ 是 $n \times n$ 矩阵, $q \in Z^{+}$, 对 $J$ 的任一特征根 $\lambda \in C$, 有

$$
\lambda \neq 1 \Rightarrow \lambda^{q} \neq 1 \text {, }
$$

则 $\sum_{i=0}^{9-1} J^{i}$ 是非退化矩阵.

证 只需验证 $\sum_{i=0}^{a-1} J^{i}$ 无零特征根, 设 $\lambda_{1}, \lambda_{2}, \cdots, \lambda_{n}$ 是 $J$ 的全部特征根, 则不难证明 $\sum_{i=0}^{a-1} J^{i}$ 相似于复三角阵

$$
\left(\begin{array}{cccc}
\sum_{i=0}^{q-1} \lambda_{i}^{i} & & & 0 \\
\ddots & \ddots & \sum_{i=0}^{g-1} \lambda_{i}^{2} & \\
& \ddots & \ddots & \sum_{i=0}^{q-1} \lambda_{i}^{i}
\end{array}\right),
$$

由此结论显然. 证毕.

引理 5 设 $f \in C^{r}\left(B_{0}, B_{0}\right)$, 则对 $\forall k \in Z^{+}, \forall \varepsilon>0, \exists \delta>0$, 使得

$$
g \in V_{f}(\delta) \Rightarrow\left\|g^{k}-f^{k}\right\|_{c^{1}}<\varepsilon \text {. 证略. }
$$

引理 6 设 $f \in C^{\prime}\left(B_{0}, B_{0}\right), X_{0} \in \operatorname{Per}(f, k), \operatorname{Orb}_{f}\left(X_{0}\right)$ 在 $B_{0}$ 内部, 则对任意 $\delta>0$, 分 别存在 $X_{0}, f\left(X_{0}\right), \cdots, f^{k-1}\left(X_{0}\right)$ 的邻域 $U_{1}, U_{2}, \cdots, U_{k} \subset B_{0}$, 及 $g \in C^{r}\left(B_{0}, B_{0}\right)$, 使得以下 三条成立:

1) $g^{i}\left(X_{0}\right)=f^{i}\left(X_{0}\right), i=1,2, \cdots, k$;

2) $\left.D g\right|_{v_{i}}=\left.D f\right|_{f^{i-1}}\left(X_{0}\right), i=1,2, \cdots, k$;

3) $\|f-g\|_{c^{1}}<\delta$. 证略.

引理 7 设 $J$ 是 $n \times n$ 矩阵, $J$ 有满足定理 1 中 $\mathrm{i})$ 和 ii) 的一组特征根, 则线性映射

$$
F: R^{n} \rightarrow R^{n}, X \mapsto J(X),
$$

在 $O$ 的任一邻域有无穷多 $m$ 周期点.

由线代数知识及文献 [7], 可直接验证此结论成立.

\section{三、定理的证明}

由引理 1, 不妨设 $M=B_{0}, X_{0}=0$, 且 $\operatorname{Orb}_{f}\left(X_{0}\right)$ 在 $B_{0}$ 内部.

证明定理 1. (1) 必要性. 记 $\Delta$ 为 $J=\left.D f^{k}\right|_{0}$ 的特征根之集，令 $\Delta_{1}=\left\{\lambda \mid \lambda^{m}=1, \lambda \epsilon\right.$ $\Delta\}$ ，反设必要条件不成立，则对 $\left.\left.\Delta_{1}, i\right) 、 i i\right)$ 不同时成立，归三种情况:

1) 当 $m>1, \Delta_{1} \neq \phi$ 时, 取 $p_{0}=\min \left\{p \in Z^{+} \mid \forall \lambda \in \Delta_{1}, \lambda^{p}-1\right\}$ 则 $p_{0}<m$ 且 $p_{0} \mid m$ 设 $m-p_{0} q$, 则 $q>1$. 因 $J^{p_{0}}$ 的特征根之集 $\Lambda$ 为 $\left\{\alpha=\lambda^{p_{0}}\{\lambda \in \Delta\}\right.$, 由 $\Delta_{1}$ 的定义, 当 $\lambda \in \Delta_{1}$

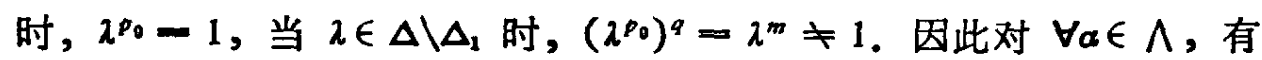




$$
\alpha \neq 1 \Rightarrow \alpha^{q} \neq 1
$$

由引理 $4, \sum_{i=0}^{q-1}\left(J^{p_{0}}\right)^{i}$ 是非退化的. 由引理 3 , 存在 $\eta_{0}>0$, 使得对 $\forall X \in R^{n}, X \neq 0$ 有

$$
\left\|\left(\sum_{i=0}^{q-1}\left(J^{p_{0}}\right)^{i}\right)(X)\right\|>\eta_{0}\|X\| \text {. }
$$

由求微分的链法则有 $\left.D f^{k p_{0}}\right|_{o}=\left(\left.D f^{k}\right|_{0}\right)^{p_{0}}=J^{p_{0}}$, 因而由引理 2, 对 (1) 中 $\eta_{0}, \exists \delta_{1}>0$, $\varepsilon_{1}>0$, 使得对 $\forall g \in V_{f}^{k p_{0}}\left(\delta_{1}\right), \forall X \in U_{o}\left(\varepsilon_{1}\right)$ 有

$$
\left\|g^{a}(X)-X-\left(\sum_{i=0}^{q-1}\left(J^{p_{0}}\right)^{i}\right)(g(X)-X)\right\| \leqslant \eta_{0}\|g(X)-X\| .
$$

如果 $X_{0} \in U_{o}\left(\varepsilon_{1}\right)$ 是 $g$ 的 $q$ 周期点, 则因 $q>1$, 有 $g\left(X_{0}\right)-X_{0} \neq 0, g^{q}\left(X_{0}\right)-X_{0}=0$, 又由 (2) 式有

$$
\left\|\left(\sum_{i=0}^{q-1}\left(J^{p_{0}}\right)^{i}\right)\left(g\left(X_{0}\right)-X_{0}\right)\right\| \leqslant \eta_{0}\left\|g\left(X_{0}\right)-X_{0}\right\| .
$$

显然 (1) 式与 (3) 式矛盾. 故对 $\forall g \in V, k^{k p_{0}}\left(\delta_{1}\right), g$ 在 $U_{o}\left(\varepsilon_{1}\right)$ 中无 $q$ 周期点, 由引理 5 , 存 在 $\delta_{1}^{\prime}>0$, 使得对 $\forall h \in V_{f}\left(\delta_{1}^{\prime}\right)$ 有 $h^{k p_{0}} \in V_{f} k p_{0}\left(\delta_{1}\right)$, 因而 $U_{O}\left(\varepsilon_{1}\right)$ 中无 $h^{k p_{0}}$ 的 $q$ 周期点, 故 $U_{0}\left(\varepsilon_{1}\right)$ 中无 $h$ 的 $k p_{0} q=m k$ 周期点. 由定义, $O$ 不是 $m$ 分歧周期点.

2) 当 $m>1, \Delta_{1}=\phi$ 时,与上之讨论一样,得同样结论.

3) 当 $m=1$ 时, 必有 $\Delta_{1}=\phi$, 即 $J-I$ 是非退化阵, 因而不难验证存在 $\delta_{2}>0$, $\varepsilon_{2}>0$, 使得对 $\forall g \in V_{f}^{k}\left(\delta_{2}\right), g-I$ 在 $U_{o}\left(\varepsilon_{2}\right)$ 上至多有一个零点, 即 $g$ 在 $U_{o}\left(\varepsilon_{2}\right)$ 上至多 有一个不动点, 因此 $O$ 不是 1 分歧周期点.

由 1), 2), 3) 必要性得证.

(2) 充分性. 设 $O \in \operatorname{Per}(f, k),\left.D f^{k}\right|_{o}$ 有满足 i) 和 ii) 的一组特征根. 任取 $\delta>0$, 由 引理 6 , 分别存在 $O, f(O), \cdots, f^{k-1}(O)$ 的互不相交的邻域 $U_{1}, U_{2} \cdots, U_{k}$ 及 $g \in C^{r}\left(B_{O}, B_{o}\right)$, 使得引理 6 中三条成立. 由连续性, 存在 $O$ 的邻域 $U_{0} \subset U_{1}$, 使得 $g^{k}\left(U_{0}\right) \subset U_{1}, g^{i}\left(U_{0}\right) \subset U_{i+1}$ $(i-1,2, \cdots, k-1)$. 易见 $\left.D g^{k}\right|_{v_{0}}-J$. 由引理 7, 在 $O$ 的任一邻域里有无穷多 $g^{k}$ 的 $m$ 周期点. 由于 $U_{1}, U_{2}, \cdots, U_{k}$ 互不相交, 故在 $O$ 的任一邻域里有无穷多 $g$ 的 $m k$ 周期点, 由 定义, $O$ 是 $t$ 的 $m$ 分歧周期点. 充分性得证.

证明定理 2. 设 $\Delta$ 为 $\left.D f^{k}\right|_{0}$ 的特征根之集. 证毕.

令 $M=\left\{m \in Z^{+} \mid \exists \lambda \in \Delta, \lambda\right.$ 为 $m$ 次单位根 $\}$, 又令 $\mathscr{A}(M)=\left\{n \in Z^{+} \mid \exists m_{1}, \cdots, m_{s} \in M\right.$, 使得 $n$ 为 $m_{1}, \cdots, m_{s}$ 的最小公倍数 $\}$. 易见, \#舟 $(M) \backslash\{1\}) \geqslant \# \mathscr{A}(M)-1$.

由于 $\Delta$ 中复特征根是以共轭对出现的, 又不等于 1 的单位根只能是 2 次单位根一 -1 , 故

$$
\#(M \backslash\{1\}) \leqslant \begin{cases}\frac{n}{2}, & \text { 当 } n \text { 为偶数. } \\ \frac{n+1}{2}, & \text { 当 } n \text { 为奇数. }\end{cases}
$$

因此 


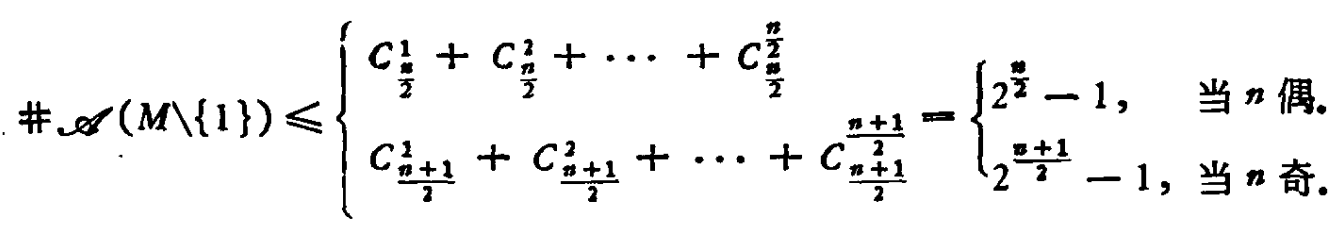

不难看出, 当 $n$ 奇, \# $\mathscr{A}(M \backslash\{1\})=2^{\frac{n+1}{2}}-1$ 时, $1 \notin M$, 而当 $1 \in M$ 时, \# $\mathscr{A}(M)$ $\left\{<2^{\frac{n+1}{2}}-1\right.$. 因此得出:

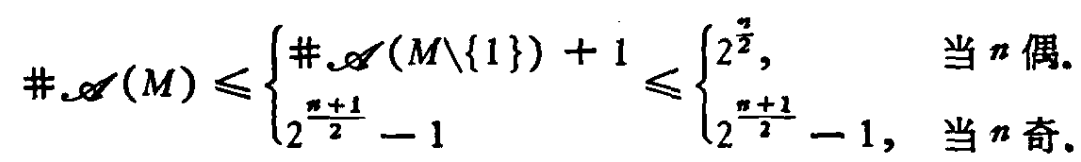

对 $\forall m \&(M)$ ，由定理 1 之推论， $m$ 不能成为 $O$ 的分歧倍数,因而得出

$$
B_{f}^{n}(O) \leqslant \# \mathscr{A}(M) \leqslant \begin{cases}2^{\frac{n}{2},} & \text { 当 } n \text { 偶. } \\ 2^{\frac{n+1}{2}}-1, & \text { 当 } n \text { 奇. }\end{cases}
$$

注 1. 定理 2 中的等式可以成立. 可见高维流形上的周期轨分歧远比一维复杂, 这就使 得对高维流形上分歧引起的非常有趣的现象比一维情形更难以从理论上进行深刻的解释. 本 文结果或许能影响这方面的研究,因为至少说明了分歧的原因.

注 2. 显然,本文结果包含了 Block 等关于线段自映射分歧倍数的结果 ${ }^{[2]}$.

致谢: 在本文写作中承蒙张景中教授和杨路教授的具体指导,在此表示哀心的感谢.

[ 1 ] Collet, P., Eckmann, J. P., Iterated Maps on the Inzerval as Dynamical Systems, Boston, Birkhäuser, 1980, $173-227$.

[2] Block, L. and Hart, D., The bifurcation of periodic orbits of one-dimensional maps, Ergod. Th. \& Dynam. Sys, 2(1982), 125-129.

[ 3 ] Collet, P., Eckmann, J. P., Lanford, O. E., Universal properties of maps on an interval. Commun. Mash. Phys, 76(1980), 211-254.

[4] 杨路、张貫中, 中国科学, A 辑, 1985, 12: 1061-1069.

[5] 郝柏林, 物理学进展, 3(1983)，3: 329-416.

[6] Lia and Yorke, Period three implies chaos, Amer. Mazh. Monshly, 82(1975), 985-992.

[7] 张筑生, 微分动力系统讲义, 1983, 149-152. 